\title{
RETRACTED: Enhanced recovery after elective cesarean sections
}

\section{Original Article}

\section{Sara Taha Mostafa}

Department of Obstetrics \& Gynecology, Faculty of Medicine, Benha University, Benha, Egypt

\begin{abstract}
Objective: To compare the early recovery after surgery (ERAS) pprotocol with the conventional one in yomentergoing elective CS.

Patients and Methods: The study included 96 women undergoing elective cesarean section for fiferent reasons They were randomly divided into two groups 48 patients each, Group (A) received the ERAS resimen and Group (B) was managed with the conventional care. Women with major medical or obstetric disorders we excluded.

Results: Cases' age ranged between 18- 35 years without significant difference between groups. Also, gestational age, haemoglobin concentration and platelet count were comparable between groups. In and pos-operative uasea and vomiting were significantly higher ( $\mathrm{p}$ value $<0.0366$ ) in the control group ( $8 \mathrm{vs}$ ). Orotv had significantly shorter interval to oral intake, ambulation, first intestinal sound and first motion. Moreo er, the need to use opiate for pain control with overall pain scores were significantly lower in study group with signifoantly better satisfaction rates and shorter hospital stay.

Conclusion: ERAS protocol for women planned for elective CS is effective in contoling perioperative gastrointestinal symptoms, pain control and encourages early ambulation with of fring earlier resumption of intestinal motility, higher satisfaction and fewer days of admission.

Key Words: Caesarean section (CS), early recovery after surgery (ERAS)

Received: $26^{\text {th }}$ April 2019, Accepted: $14^{\text {th }}$ June 2019

Corresponding Author: Sara Taha Mostafa, Department of Obstetrics \& Gynecology, Benha University Hospitals, Elsaha Street of Fareed Nada Street, Benha, Qalubeya Egypt, Tl,00201226401231, E-mail: drsarataha75@yahoo.com
\end{abstract}

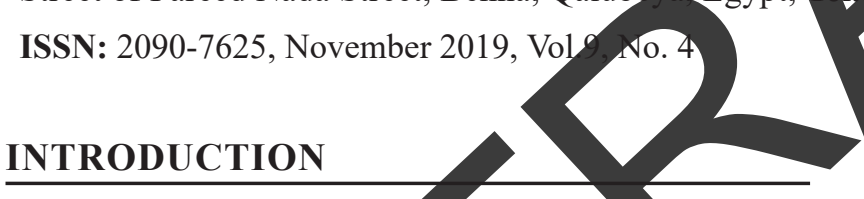

One of the most comm jor surgeries in the world is Many nations, proor exsts that an increasing percentage of all CSs account for scheduled or elective surgeriest. Despite initiatives to counter this trend, the rate of optional es continues to grow. CS birth is linked to long hospitalizan compared with spontaneous and bulk of fenales had to remain the hospital for a mininum of two days post a scheduled CS operation ${ }^{[2]}$. The handing of CS and postoperative care placed a significant straim on the countries' care and costs. Most of the females in these surgeries are young and fit. They not only have the ability to recover rapidly, but the birth of a fresh child is a distinctive motive to return to ordinary function rapidly. Hospital discharge could possibly halve stay for this women group after the operation and thus significantly reduce care burden and enhance savings for different obstetrical units ${ }^{[3]}$.

The introduction of enhanced recovery (ER) for the scheduled CS to promote early discharge is common. After elective operation, the idea of an enhanced recovery program is not fresh ${ }^{[4]}$. The purpose of improved recovery is to optimize various patient care elements so that rehabilitation can be improved to facilitate earlier release without decline in patient satisfaction or care quality ${ }^{[5]}$.

The broad acceptance of such management is undoubtedly connected with increasing proof of advantages such as decreased maternal morbidities, shorter hospital admission time and quick return to ordinary daily routine for cases used these improved rehabilitation programs ${ }^{[6]}$. The National Institute of Health and Care Excellence suggested in UK women recovering well should be freed soon from the hospital (after 24 hours) and followed-up at their homes as this was not connected with further rise of infant or maternal readmissions.

Strategies of ER protocols included trials of the following; good review of pre-conception information to future mothers, proper perioperative hydration as well as nutrition, implementation of minimally invasive surgical techniques wherever applicable, maintain normothermia, avoid postoperative gastrointestinal upset, early urinary catheter removal, adequate pain relief and early postoperative mobilization. 